EPJ Web of Conferences 32, 02002 (2012)

DOI: $10.1051 /$ epjconf/20123202002

(C) Owned by the authors, published by EDP Sciences, 2012

\title{
Energetic Particle Physics Experiments With High Power ECRH on HL-2A
}

\author{
X.T.Ding, W.Chen, L.M.Yu, S.Y.Chen*, J.Q.Dong, X.Q.Ji, Z.B.Shi, Y.Zhou, Y.B.Dong, \\ X.L.Huang, Z.W.Xia, X.Y.Song, X.M.Song, J.Zhou, J.Rao, M. Huang, B.B.Feng, Y.Huang, Yi. \\ Liu, L.W.Yan, Q.W. Yang, X.R.Duan and HL-2A experiment team \\ Southwestern Institute of Physics, Chengdu 610041 \\ *College of Physical Science and Technology, Sichuan University, Chengdu 610065
}

\begin{abstract}
The MHD instabilities driven by the energetic-particles are of particular importance for future burning plasma devices, where energetic particles will be abundantly produced by high power heating and fusion reaction. In this paper, some new phenomena induced by high power ECRH are presented.

The high frequency $(200 \mathrm{kHz}-350 \mathrm{kHz})$ instabilities have been observed during the high power ECRH or NBI. The frequencies of the modes are proportional to Alfvén velocity and very close to the TAE frequency in the plasma core. In the lower frequency range from $10 \mathrm{kHz}$ to $35 \mathrm{kHz}$, the coexistence of multi-mode has also been found during the high power ECRH. The frequencies of the modes are proportional to Alfvén velocity, but much lower than it. Between the two frequency ranges, a new mode with the frequency from $50 \mathrm{kHz}$ to $100 \mathrm{kHz}$ has been observed with high power ECRH and NBI heating together. The modes have obvious frequency chirping within short time, which is the feature of the EPM like modes.

The fishbone instability excited by energetic electrons during ECRH has been identified in HL-2A. It is interested to find the frequency jump phenomena in the high power ECRH. The frequency jumps between $8 \mathrm{kHz}$ and $15 \mathrm{kHz}$ within about $25 \mathrm{~ms}$ periodically, when the power is $1.2 \mathrm{MW}$. The new results are different from the phenomena on Tore-Supra, because the trapped particle dominates in this case.
\end{abstract}

\section{Introduction}

To study the interaction of the energetic particles with collective modes, including the toroidal Alfvén eigenmode (TAE) and the energetic particle modes (EPM), is very important for the burning plasmas. These instabilities excited by fast ions from high power heating or fusion reaction. The drive for these modes depends on the energy of the particles rather than the mass, so that energetic electrons can also drive these unstable modes. Understanding the interaction between the energeticelectrons and these modes can provide a contribution for burning plasma research, because their effect on low-frequency MHD modes can be used to simulate and analyze the alpha particles characterized by small dimensionless orbits, which are similar to energetic-electrons in the middle size tokamak plasma ${ }^{[1]}$. The interaction of the energetic electrons with collective modes may play an important role in the plasma with high electron temperature and low collisionality in fusion reactor. Indeed, the processional drift resonance was first described for fast electrons ${ }^{[2]}$. Fast electron driven TAEs were first seen on Compass-D with combined electron cyclotron heating $(\mathrm{ECH})$ and lower

This is an Open Access article distributed under the terms of the Creative Commons Attribution License 2.0, which permits unrestricted use, distribution, and reproduction in any medium, provided the original work is properly cited. 
hybrid current drive (LHCD) ${ }^{[3]}$. The fishbone instability driven by fast electrons has been observed with electron cyclotron current drive (ECCD) on DIII-D ${ }^{[4]}$ and with ECRH on HL-1M ${ }^{[5]}$, and also with LHCD on FTU ${ }^{[1]}$ and Tore Supra ${ }^{[6]}$. Recently, the Beta induced Alfvén eigenmode (BAE) excited by energetic electrons has been found and identified in the experiments on the HL-2A tokamak ${ }^{[7]}$. Table1 list the experimental observations about the instabilities driven by energetic electrons in the different fusion devices.

Table: instabilities driven by energetic-electrons in different fusion devices

\begin{tabular}{|c|c|c|c|c|c|}
\hline Device & Heating and power & Instability type & Mode character & Energy of EE & Reference \\
\hline DIII-D & ECRH, $\sim 1.1 \mathrm{MW}$ & fishbone & $\begin{array}{l}\mathrm{m} / \mathrm{n}=\mathrm{l} / 1 \\
\mathrm{f} 10 \mathrm{kHz}\end{array}$ & $\begin{array}{c}\text { No } \\
\text { measurement }\end{array}$ & PRL2000 \\
\hline Compass-D & $\mathrm{ECRH},<1.3 \mathrm{MW}$ & Like EPM & $\begin{array}{c}m / n=1 / 1 ? \\
f \sim 300-500 \mathrm{kHz}\end{array}$ & $10-100 \mathrm{keV}$ & NF2000 \\
\hline HL-1M & $\operatorname{ECRH}(\bullet 0.25)+\mathrm{LHW}(-0.16 \mathrm{MW})$ & fishbone & $\begin{array}{l}\mathrm{m} / \mathrm{n}=\mathrm{l} / \mathrm{l} \\
\mathrm{f} \sim 6-8 \mathrm{kHz}\end{array}$ & $\begin{array}{c}\text { No } \\
\text { measurement }\end{array}$ & NF2002 \\
\hline FTU & $\mathrm{LHW},<2.5 \mathrm{MW}$ & fishbone & $\begin{array}{c}\mathrm{m} / \mathrm{n}=\mathrm{l} / 1 \\
\mathrm{f} \sim 5-15 \mathrm{kHz}\end{array}$ & $20-150 \mathrm{keV}$ & $\begin{array}{l}\text { NF2007 } \\
\text { NF2010 }\end{array}$ \\
\hline Tore-Supra & $\mathrm{LHW},<3.0 \mathrm{MW}$ & fishbone & $\begin{array}{c}\mathrm{m} / \mathrm{n}=1 / 1,2 / 2,3 / 3 \\
\mathrm{f} \sim 6-12 \mathrm{kHz}, \\
\text { freq. jumps }\end{array}$ & $20-80 \mathrm{keV}$ & $\begin{array}{l}\text { NF2006 } \\
\text { PRL2009 }\end{array}$ \\
\hline C-Mod & $\mathrm{LHW},<1.0 \mathrm{MW}$ & TAE & $\mathrm{n}=1-6, \mathrm{f}-200-800 \mathrm{kHz}$ & $40-100 \mathrm{keV}$ & NF2008 \\
\hline HSX & $\mathrm{ECRH},<0.4 \mathrm{MW}$ & Like acoustic & $\begin{array}{l}\mathrm{m} / \mathrm{n}=3 / 1,5 / 1 ?, \\
\mathrm{f}-20-120 \mathrm{kHz}\end{array}$ & $\begin{array}{c}\text { No } \\
\text { measurement }\end{array}$ & PRL2009 \\
\hline CHS & $\mathrm{ECRH}, \sim 0.1 \mathrm{MW}$ & EPM & $\mathrm{m} / \mathrm{n}=2 / 1, \mathrm{f} \sim 50-100 \mathrm{kHz}$ & $\begin{array}{c}\text { No } \\
\text { measurement }\end{array}$ & NF2010 \\
\hline HL-2A & ECRH, $0.4-1.5 \mathrm{MW}$ & $\begin{array}{l}\text { Fishbone, } \\
\text { BAE }\end{array}$ & $\begin{array}{c}\mathrm{m} / \mathrm{n}=1 / 1, \mathrm{f}-5-15 \mathrm{kHz} \\
\mathrm{m} / \mathrm{n}=3 / 1,10-25 \mathrm{kHz}\end{array}$ & $30-70 \mathrm{keV}$ & $\begin{array}{l}\text { NF2009 } \\
\text { NF2010 } \\
\text { PRL2010 }\end{array}$ \\
\hline
\end{tabular}

In this paper, the new experimental results about the energetic particle physics during high power ECRH will be presented. It is interesting to study the features of the observed modes, because their behaviors may be related with the energetic particle losses in the future fusion reactor.

\section{HL-2A tokamak and high power ECRH system}

HL-2A is a medium-size tokamak ${ }^{[8]}$, with the major radius $\mathrm{R}=1.65 \mathrm{~m}$ and minor radius $\mathrm{a}=0.4 \mathrm{~m}$, which performs with the plasma current $\mathrm{Ip}=100 \mathrm{kA}-350 \mathrm{kA}$, the toroidal magnetic field $\mathrm{Bt}$ in the range 1T-2.5T and line-averaged electron density $\left\langle\mathrm{n}_{\mathrm{e}}\right\rangle=(0.3-5) \times 10^{19} \mathrm{~m}^{-3}$. To investigate the energetic electron behaviors and their interaction with the collective modes, it is operated with low plasma density and the high power ECRH. In these discharges, the electron can be accelerated both by the ohmic electric field and ECRH. The fast electrons are of asymmetric velocity distributions in the different discharges. The high power ECRH system has been installed with six $68 \mathrm{GHz} / 500 \mathrm{~kW} / 1 \mathrm{~S}$ gyrotrons. The maximum injecting power is up to $2.4 \mathrm{MW}$, after taking off the transmission loss. Both fundamental O-mode and second harmonic X-mode are adopted and inject to plasma from the low field side (LFS). The ECW power can deposit at different radius both in LFS and HFS through change the toroidal magnetic field.

In the HL-2A tokamak there are about 30 diagnostics to provide the general plasma parameters, such as electron temperature, ion temperature and electron density. The features of the collective modes are observed by Mirnov probes and soft x-ray detector arrays. The Mirnov probe arrays are arranged around the wall of the device with 18 coils poloidlly and 10 coils toroidally. Five soft x-ray detector arrays are of 100 channels totally. The spatial and temporal resolutions are $2.5 \mathrm{~cm}$ and 10 microseconds, respectively. Cadmium-telluride ( $\mathrm{CdTe}$ ) detector is used to monitor the hard X-ray 
(HXR) in the energy range of $10-200 \mathrm{keV}$, which can be divided to 10 energy ranges. The nonthermal radiation measured by the ECE is also used to analyze the behaviors of the energetic electrons.

\section{Experimental results}

\subsection{Review of the physics phenomena induced by energetic electrons on HL-2A}

The fishbone has been experimentally observed during ECRH on the HL-2 $\mathrm{A}^{[9,10]}$. It is also found that the energetic electrons, which deviate from Maxwell velocity distribution, excite the mode, therefore, called e-fishbone. The energy distribution of the electrons is measured by the hard x-ray detector $(\mathrm{CdTe})$ with the pulse height analysis. Experiments show that energetic electrons with 35$70 \mathrm{keV}$ play important role to drive the mode. The e-fishbone can be excited during off-axis ECRH deposited on both the high field side and the low field side. In order to identify the e-fishbone mode, the resonance condition of the wave-particle has been investigated. Comparing with experimental results, the calculation analyses show that the mode frequency is close to the precession frequency of the barely trapped electrons or barely circulating electrons when the magnetic shear is very weak or negative. With increasing of the ECRH power, the e-fishbone frequency increases, and shows some new phenomena with up- and down-chirping behaviors, and sometimes also with V-font-style sweeping.

Another low frequency MHD mode excited by energetic electrons has been observed for the first time both in the Ohmic and electron cyclotron resonance heating plasma in HL-2A ${ }^{[7]}$. The mode frequency is comparable to that of the continuum accumulation point of the lowest frequency gap induced by the shear Alfvén continuous spectrum due to finite Beta effect, and it is proportional to Alfvén velocity at thermal ion- Beta held constant. Therefore, the mode can be identified as Beta induced Alfvén eigenmode (e-BAE). The experimental results show that the BAE is related not only with the population of the energetic electrons, but also their energy and pitch angles. The results indicate that the barely circulating and deeply trapped electrons play an important role in the mode excitation.

With the increasing of the ECRH power, the abundant unstable modes in different frequency range can be observed on HL-2A. In the following sections, some new experimental phenomena will be presented.

\subsection{The TAE during ECRH}

The high frequency $(200 \mathrm{kHz}-350 \mathrm{kHz})$ instabilities have been observed during the high power ECRH heating in the HL-2A tokamak. It also can be observed with medium power NBI heating. These high frequency modes have relatively small amplitude measured by the Mirnov pick-up coils on wall. The typical discharge is shown in the Figure 1. 



Fig. 1 (a) the line averaged electron density detected by a HCN laser interferometer; (b) the magnetic fluctuations measured by Mirnov probe; and (c) the frequency spectrum of the magnetic fluctuation from the up to the bottom.

In this discharge $\left(\mathrm{B}_{\mathrm{T}}=1.3 \mathrm{~T}, \mathrm{Ip}=130 \mathrm{kA}\right)$ the high power ECRH $(1.8 \mathrm{MW})$ and the NBI heating $(535 \mathrm{~kW})$ have been added in the different time durations: ECRH from $400 \mathrm{~ms}$ to $620 \mathrm{~ms}$, NBI from $620 \mathrm{~ms}$ to $1120 \mathrm{~ms}$. Figure 1 show (a) the line averaged electron density detected by a HCN laser interferometer; (b) the magnetic fluctuations measured by Mirnov probe; and (c) the frequency spectrum of the magnetic fluctuation from the up to the bottom. During the ECRH the density decreases because of the pump out effect and it becomes high with high power NBI. The magnetic fluctuations can be measured by Mirnov probes both in ECRH and NBI heating. These modes disappear in the high density during ECRH, indicating the energetic electrons play an important role to excite the modes. It can be found that the frequency of the magnetic fluctuations increases with decreasing of the density, as shown in Fig. (c) At the same time, the frequency is modulated by gas puffing: after the gas puffing the frequency decreases, and then increases up to next puffing which mean that the frequency is the function of the plasma density. As we know, the Alfvén velocity is proportional to $B n_{e}^{-1 / 2}$, here $B$ is magnetic field and $n_{e}$ is plasma density, so it is important to check the relation between the frequencies and $B n_{e}{ }^{-1 / 2}$. Fig 2 shows the statistical curves for frequency of the modes versus the $\mathrm{B} \mathrm{n}_{\mathrm{e}}^{-1 / 2}$ with the magnetic field from $1.2 \mathrm{~T}$ to $1.4 \mathrm{~T}$ and the line averaged electron density from $0.7 \times 10^{19} \mathrm{~m}^{-3}$ to $2.3 \times 10^{19} \mathrm{~m}^{-3}$. The curves show clearly that the frequencies of the modes are proportional to Alfvén velocity, indicating that they are related with Alfvén mode. 


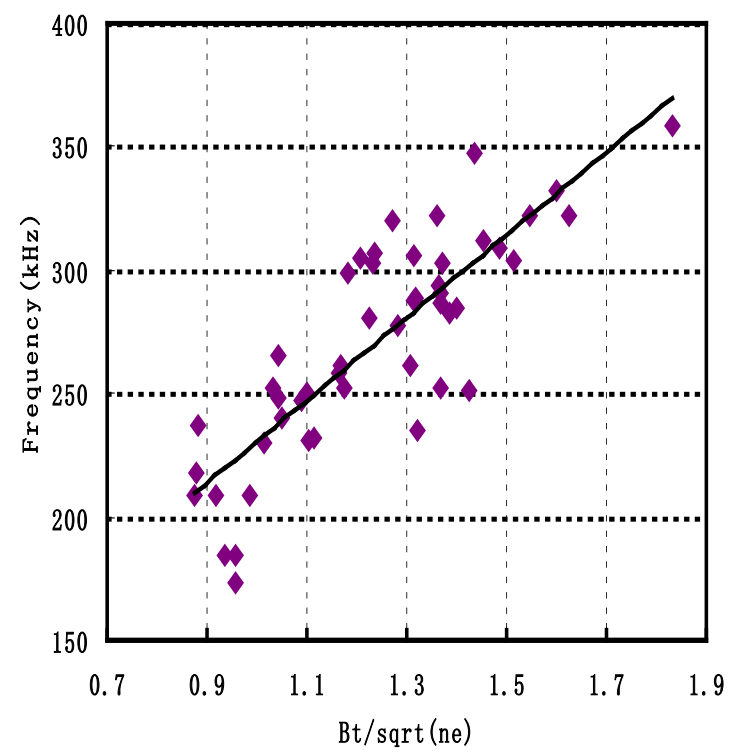

Fig.2 The frequency of the mode versus the $B n^{-1 / 2}$ with the magnetic field from $1.2 T$ to $1.4 T$ and the line averaged electron density from $0.7 \times 10^{13} \mathrm{~cm}^{-3}$ to $2.3 \times 10^{13} \mathrm{~cm}^{-3}$.

These high frequency modes have relatively small amplitude, so it is difficult to be measured by soft x-ray or ECE, because of the higher noise. Therefore their local position and mode numbers can not be determined. To compare with the toroidal Alfvén eigenmode (TAE), the frequency of the $\mathrm{TAE}$ at the different radius has been estimated. As we know that the frequency of TAE is

$$
f_{T A E} \cong v_{A} /\left(4 \pi q_{T A E} R\right)
$$

Here, $\mathrm{V}_{\mathrm{A}}$ is Alfvén velocity, $\mathrm{q}_{\mathrm{TAE}}$ is safety factor and $\mathrm{R}$ is major radius. In the plasma core, the $\mathrm{q}$ $\sim 1$, the density is about $1-3 \times 10^{19} \mathrm{~m}^{-3}$, then the frequency could be $180 \mathrm{kHz}-330 \mathrm{kHz}$ according to above formula. The frequency is very close to the experimental results, as shown in Fig. 2. In fact, the neutral beam with $40 \mathrm{keV}$ can be injected easily to plasma center and drives TAE. Experiments show that the energetic electrons produced by high power ECRH can also play a same role to drive the TAE in plasma core. It is easy to estimate the frequency of TAE in other q $>1$ surfaces, with the hyperbolic density profile. In general, the frequency is from $120 \mathrm{kHz}$ to $250 \mathrm{kHz}$. Therefore, the observed modes are also possible to be in these areas.

\subsection{Lower frequency MHD modes during ECRH}

The low frequency modes can be observed in high power ECRH plasmas with the plasma parameters: $\mathrm{Ip}=155 \mathrm{kA}-160 \mathrm{kA}, \mathrm{Bt}=1.2 \mathrm{~T}-1.4 \mathrm{~T}$ and line averaged electron density $<\mathrm{n}_{\mathrm{e}}><1.4 \times 10^{19} \mathrm{~m}^{-}$ ${ }^{3}$. These modes disappear in the high density discharges, indicating the energetic electrons are important to excite the modes. To observe the features of these modes in the different heating power, the ECRH power is increased step by step. The typical waveforms are shown in Figure 3: (a) plasma current, (b) the line averaged electron density detected by a HCN laser interferometer; (c) electron temperature from ECE, (d) hard X-ray (HXR), (e) the magnetic fluctuations measured by Mirnov probe; (f) the ECRH power; ( $g$ ) the frequency spectrum of the magnetic fluctuation from the up to 
the bottom. Since the toroidal magnetic field is $1.33 \mathrm{~T}$ in this shot, so the ECRH power deposits at the minor radius of $\mathrm{r}=15 \mathrm{~cm}$ in the lower field side mainly.

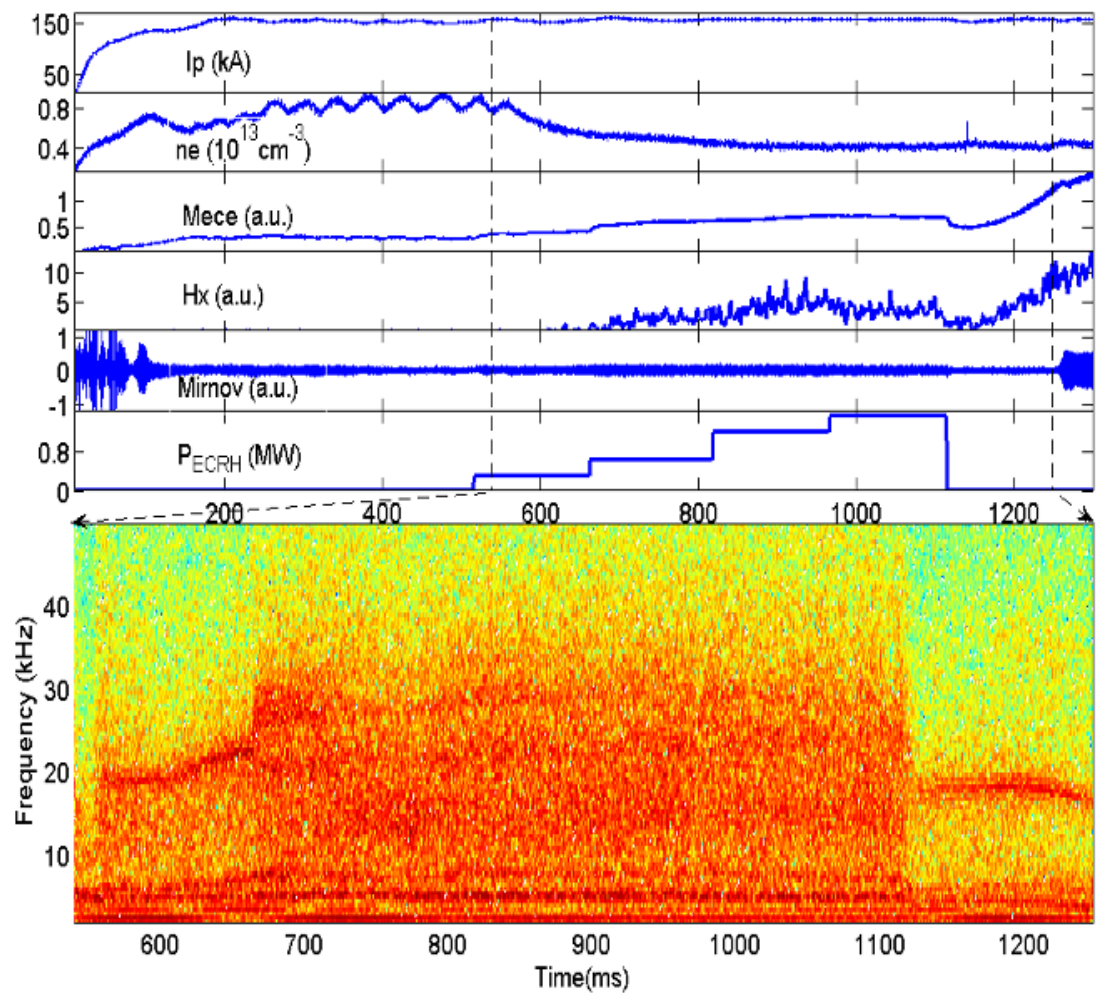

FIG.3. Evolution of (a) the plasma current, (b) the line averaged electron density; (c) electron temperature from ECE, (d) hard X-ray (HXR) in the energy range of 10-20keV (e) the signal of Mirnov probe; (f) the ECRH power; (g) the frequency spectrum of Mirnov signal in shot 17461

From Fig.3 we can see that the electron temperature measured by ECE increases with ECRH power and the electron density keeps a constant. The HXR emitted by energetic electrons increases with the ECRH power dramatically. The evolution of the frequency spectrum of the magnetic fluctuation measured by Mirnov probes is shown in the Fig.3 (g). In the low power ECRH plasma, a peak about $22 \mathrm{kHz}$ can be seen clearly in the frequency spectrum, which has been identified as bata induced Alfvén eigenmode (e-BAE). The main characters of the e-BAE have been described in the reference [7]. When the power is over about $600 \mathrm{~kW}$, two peaks or three peaks appear, as shown in Fig 4(a). The spectrum becomes wide when the power is high enough, generally more than $1.5 \mathrm{MW}$, as shown in Fig 4(b). After ECRH switch-off, the spectrum comes back to previous one with only one frequency peak. The signals of the edge channels of the soft x-ray array show the similar frequency spectra with the Mirnov probes. The spectra disappear or are very weak in center channels, which mean that the modes are located outside of the plasma center. 


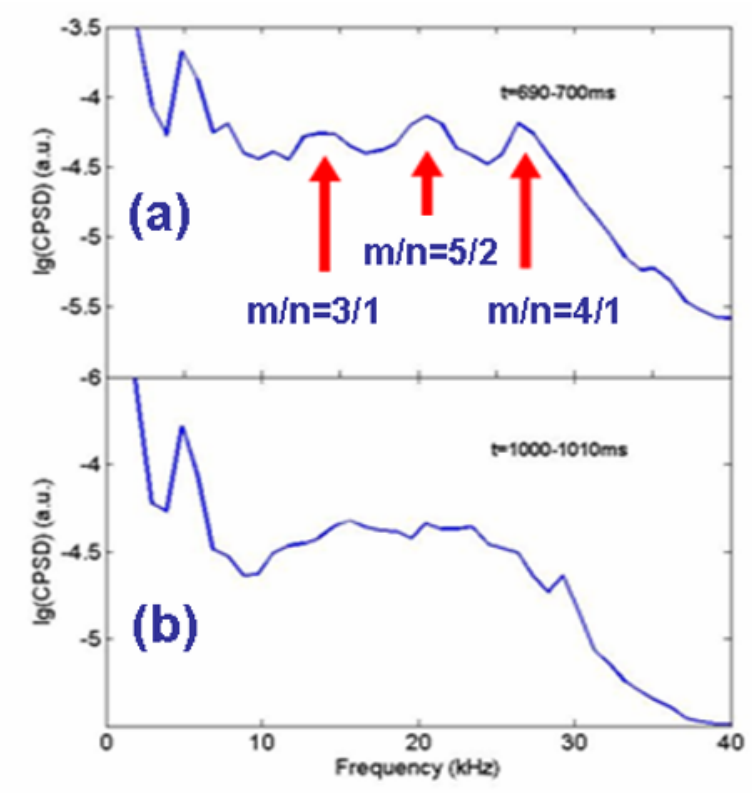

\section{Fig.4 The spectra of the magnetuc fluctuationduring different ECRH power (a) $0.6 M W$ and (b) $1.5 M W$ in shot 17461}

To obtain the detail features of the modes in high power heating, the mode numbers, the power density spectra and the correlation spectra both toroidally and poloidally have been analyzed. Three clear frequency peaks at $12.5 \mathrm{kHz}, 19.5 \mathrm{kHz}$ and $27.5 \mathrm{kHz}$ can be found in the spectra, which are denoted $f_{1}, f_{2}$ and $f_{3}$ in the figures $4(a)$. The mode numbers of the three peaks are $m / n=3 / 1,5-6 / 2$ and $4 / 1$, respectively.

It is also found that the frequencies of the three modes change with plasma density and the toloidal magnetic field. Figure 5 shows the statistical curves for frequency of the three modes versus the $\mathrm{B} \mathrm{n}_{\mathrm{e}}{ }^{-1 / 2}$ with the magnetic field from $1.2 \mathrm{~T}$ to $1.4 \mathrm{~T}$ and the line averaged electron density from $0.3 \times 10^{19} \mathrm{~m}^{-3}$ to $1.4 \times 10^{19} \mathrm{~m}^{-3}$. The curves show clearly that the frequencies of the three modes are proportional to Alfvén velocity. The toroidal Alfvén frequency can also be estimated by the formula (1). To compare the frequency of the modes with the toroidal Alfvén frequency, the local density and $q$ value have been estimated. The local density can be obtained from microwave reflectomerty at the plasma edge and 8-channel HCN laser interferometer in the plasma center. The measurement of the q-profile is not available in HL-2A, so we estimate its profile with the tokamak simulation code (TSC) ${ }^{[11]}$. The $\mathrm{q}=1$ radius can also estimate by the saw-tooth inversion radius and the edge $\mathrm{q}$ value can be calculate using plasma current, toroidal field, minor radius and major radius directly, which are agreement in the q-profile from code TSC. According to the q-profile, we can obtain the positions of the $\mathrm{q}=4,3$ and 2 , and then obtain the local densities in different q- surfaces. In shot 17929 , the local density is about $8 \times 10^{18} \mathrm{~m}^{-3}, 5.5 \times 10^{18} \mathrm{~m}^{-3}$, and $1 \times 10^{18} \mathrm{~m}^{-3}$ for $\mathrm{q}=2.5,3$ and 4 , respectively. We can calculate the Alfvén frequency of the different modes: the frequency range of the modes is from $196 \mathrm{kHz}$ to $347 \mathrm{kHz}$. Therefore, the frequencies of the three modes in figure 3 are about $10 \%$ of the toroidal Alfvén frequency, which are in the BAE and BAAE frequency gap. The nature of the modes will be identified with simulation in the future. 




Fig.5 The statistical curves for frequency of the three modes versus the $B$ ne $e^{-1 / 2}$ with the magnetic field from $1.2 T$ to $1.4 T$ and the line averaged electron density from $0.3 \times 1013 \mathrm{~cm}-3$ to $1.4 \times 1013 \mathrm{~cm}-3$. f1,f2 and $f 3$ express the three peaks of the frequency in the spectra, respectively.

\subsection{EPM like modes during NBI and ECRH}

In the previous sections, the instabilities with high frequency from $200 \mathrm{kHz}$ to $330 \mathrm{kHz}$ and low frequency from $10 \mathrm{kHz}$ to $35 \mathrm{kHz}$ have been presented. Both of them are produced by the energetic electrons during high power ECRH. In this section, another instability driven by ECRH and NBI together will be shown. This is a new mode with the frequency from $50 \mathrm{kHz}$ to $150 \mathrm{kHz}$, which is not in the TAE and BAE gap.

It is very interesting to observe the evolution of the magnetic fluctuation during ECRH and NBI heating. Figure 6 shows (a) the ECRH power; (b) the NBI power, (c) plasma current, (d) the line averaged electron density; (e) the magnetic fluctuations measured by Mirnov probe; (f) the frequency spectrum of the magnetic fluctuation from the up to the bottom. It should be noticed that the frequency spectrum of the magnetic fluctuation has been expanded from $630 \mathrm{~ms}$ to $710 \mathrm{~ms}$, in which both ECRH and NBI have been switch on. A very clear mode can be observed from $679 \mathrm{~ms}$ to $697 \mathrm{~ms}$. It is also can be found from soft x-ray in center channels, indicating that the mode located inside of the 0.5 of normalized minor radius. The mode can not be observed only with ECRH or NBI. As shown in the spectrum, the frequency of the mode decreases from $100 \mathrm{kHz}$ to about $60 \mathrm{kHz}$ with the increasing of the plasma density. 


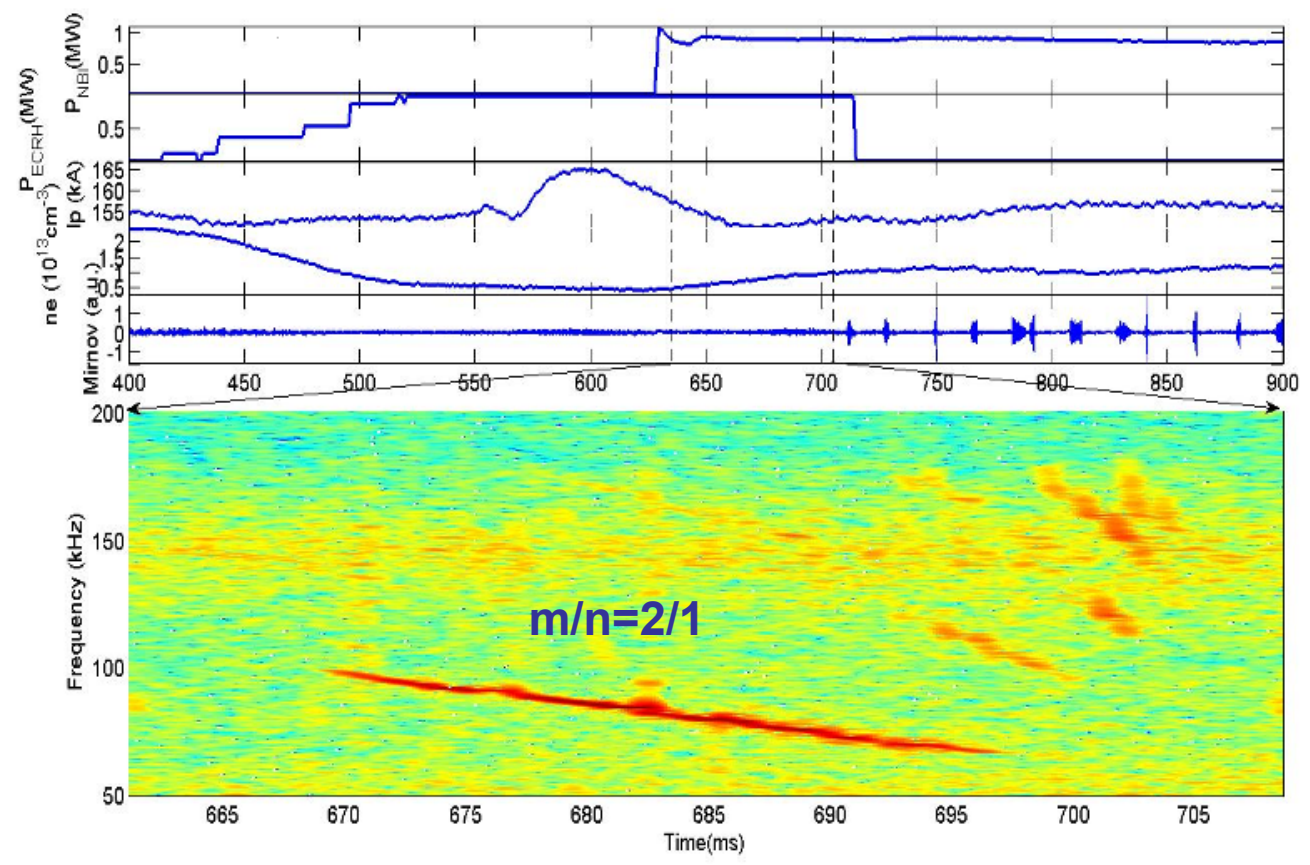

Fig. 7 (a) the ECRH power; (b) the NBI power, (c) plasma current, (d) the line averaged electron density detected by a HCN laser interferometer; (e) the magnetic fluctuations measured by Mirnov probe; (f) the frequency spectrum of the magnetic fluctuation from the up to the bottom.

The mode numbers, the power density spectra and the correlation spectra both toroidally and poloidally have been analyzed. This mode is strong collective mode with poloidal mode number $\mathrm{m}=2$ and toroidal mode number $\mathrm{n}=1$. To compare the mode frequency with TAE, the TAE frequency at the $\mathrm{q}=2$ surface has been estimated. In this discharge, $\mathrm{B}_{\mathrm{T}}=1.3 \mathrm{~T}$, the line averaged electron density increases from $0.5 \times 10^{19} \mathrm{~m}^{-3}$ to $1 \times 10^{19} \mathrm{~m}^{-3}$. Therefore, the TAE frequency at the $\mathrm{q}=2$ surface should be $150 \mathrm{kHz}$ to $220 \mathrm{kHz}$, which is much higher than the frequency of the observed $\mathrm{m} / \mathrm{n}=2 / 1$ mode. It should be noticed that there are several modes appear after $695 \mathrm{~ms}$. These modes are also excited by ECRH and NBI together and have strong chirping down frequency features. Because these modes are relative weak, their mode numbers have not been obtained yet.

Three important features of the unstable modes indicate that the modes may be energetic particle modes (EPM). At first, the frequency of the modes is between the TAE and BAE, which means that the modes are not in the gap of the continuous spectra damping of the Alfvén eigenmodes. Secondly, the obvious frequency chirping appears within short time, which is different from the Alfvén eigenmodes. Lastly, the modes are driven by very high power heating. Generally, the total heating power is about $2 \mathrm{MW}$ to drive the modes. In HL-2A the maximum NBI power and the ECRH power is about $1.2 \mathrm{MW}$ and $2.4 \mathrm{MW}$, respectively. But in the most of the discharges, the NBI power is less then $1 \mathrm{MW}$ and the ECRH power is less then 1.5MW. Therefore, the modes have not been observed only using NBI or ECRH, indicating that the low heat power is not enough to overcome the strong damping. It can explain why the modes always occur with NBI and ECRH together. This experiment seems to indicate that the EPM could be excited by both energetic ions and energetic electrons. 


\subsection{Frequency jump phenomena of e-fishbone during ECRH}

The e-fishbone has been identified and investigated during low power ECRH on HL-2A ${ }^{[9]}$. It is evident that without ECRH power, the soft x-ray signals show no fishbone activity at all, but only sawtooth activity. Hence ECRH power is the important condition for the excitation of e-fishbone activity. When the ECRH power exceeds $0.7 \mathrm{MW}$, the features of e-fishbone are complex as shown in the previous investigation in the device ${ }^{[9]}$. Periodic mode frequency jumps have been detected for the first time during high power ECRH on HL-2A.

The frequency spectra of the soft $\mathrm{x}$-ray show that the frequency of the fluctuations is about 5 $\mathrm{kHz}$ in the low ECRH power and increases to about $8 \mathrm{kHz}$ when the power increases to 1.2MW. To observe the e-fishbone behaviors during the different ECRH power, the ECRH power is added step by step from $300 \mathrm{KW}$ to $1.2 \mathrm{MW}$ in shot 17892 . The typical waveforms are shown in Figure 8 : (a) plasma current, (b) the line averaged electron density detected by a HCN laser interferometer; (c) electron temperature from ECE, (d) hard X-ray (HXR) in the energy range of 10-200keV, (e) the soft $\mathrm{x}$ ray intensity of one channel; (f) the ECRH power; ( $\mathrm{g}$ ) the frequency spectrum of the soft $\mathrm{x}$ ray fluctuation from the up to the bottom. ECE and hard X-ray emissions show that the energetic electrons increase greatly when ECRH power is higher then $0.7 \mathrm{MW}$. The frequency jump can be observed, when ECRH power increases to about $0.7 \mathrm{MW}$ and the difference from the low frequency to high frequency increases with ECRH power. The frequency jumps between $8 \mathrm{kHz}$ and $15 \mathrm{kHz}$, when the power is 1.2MW, as shown in Fig.8 (g). The frequency jump phenomena appear within about $25 \mathrm{~ms}$ periodically. The evidence of the changes of poloidal wave number can be obtained by the results of the tomography of two soft x-ray arrays. The results show that the modes are located $\mathrm{q}=1$ surface and poloidal wave numbers are $\mathrm{m}=1$ or 2 , as shown in Fig. 9. That means the mode changes between $m / n=1 / 1$ and $m / n=2 / 2$.



Fig.8 the typical temporal evolution of (a) the plasma current, (b) the plasma density, (c) the electron temperature from ECE, (d) the hard-x ray with the energy of $10-200 \mathrm{eV}$ (e) the soft x-ray intensity (f) the ECRH power and $(\mathrm{g})$ its frequency spectra in shot 17892. 

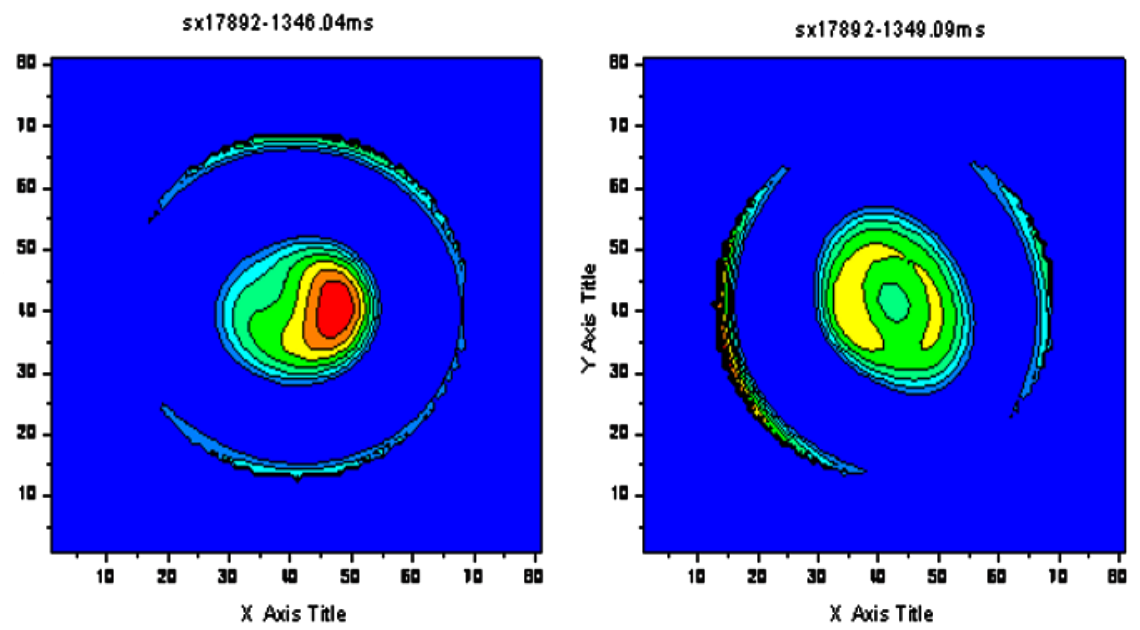

Fig.9. The evidence of the changes of poloidal wave number obtained by the results of the tomography of two soft $x$ ray arrays

These experiments show the main features of the e-fishbone during high power ECRH. The frequency jump phenomena are thought to be excited by redistribution of the energetic electrons. In Tore Supra the distributions of the energetic electrons have been obtained by the multi-channel hard $\mathrm{x}$-ray. There are only 4 channels of the hard x-ray in HL-2A, so it is impossible to measure the distribution of the energetic electrons. The multi-channel hard $\mathrm{x}$-ray system will be developed in the device. The theories have indicated that e-fishbone instabilities can be driven by both the passing particles and trapped particles. With ECRH the trapped particles are dominated. It will be very interesting to compare with the e-fishbone features during LHCD, in which the passing particles are dominated.

\section{Summary}

With the high power ECRH, the abundant new phenomena related energetic particle physics have been observed in the HL-2A tokamak. These unstable modes have been proved to be excited by energetic electrons induced by high power ECRH or together with the energetic ions.

The high frequency $(200 \mathrm{kHz}-350 \mathrm{kHz})$ instabilities have been observed during the high power ECRH or NBI heating in the HL-2A tokamak. The frequencies of the modes are proportional to Alfvén velocity and very close to the toroidal Alfvén eigenmode in the plasma core. The experiments show that the energetic electrons produced by high power ECRH can drive the TAE in plasma core.

The multi-mode coexistence phenomena occur in low frequency range from $10 \mathrm{kHz}$ to $35 \mathrm{kHz}$ during the high power ECRH. The modes located outside $20 \mathrm{~cm}$ the minor radius. When the power increases to about $0.6 \mathrm{MW}$, three modes can be observed clearly in the spectra. The mode number of the three modes is estimated to $\mathrm{m} / \mathrm{n}=3 / 1,5-6 / 2$ and $4 / 1$. The frequencies of the modes are proportional to Alfvén frequency, but much lower than it .The frequency spectra are broadening and overlap each other, when the ECRH power high enough.

A new mode with the frequency from $50 \mathrm{kHz}$ to $100 \mathrm{kHz}$ has been observed with high power ECRH and NBI together and identified as EPM like modes. The frequency of the modes is between the TAE and BAE. The obvious frequency chirping appears within short time, which is different 
from the Alfvén eigenmodes. The modes have not been observed only using NBI or ECRH, indicating that the heat power lower then $2 \mathrm{MW}$ is not enough to overcome the strong continuous spectra damping. This experiment indicates that the EPM could be excited by both energetic ions and energetic electrons.

The frequency jump of the e-fishbone can be observed, when ECRH power increases to about $0.7 \mathrm{MW}$ and the difference from the low frequency to high frequency increases with ECRH power. The frequency jumps between $8 \mathrm{kHz}$ and $15 \mathrm{kHz}$, when the power is $1.2 \mathrm{MW}$. The frequency jump phenomena appear within about $25 \mathrm{~ms}$ periodically. The modes are located $\mathrm{q}=1$ surface and the mode changes between $\mathrm{m} / \mathrm{n}=1 / 1$ and $\mathrm{m} / \mathrm{n}=2 / 2$.

\section{Acknowledgement}

The authors would like to thank the HL-2A team for tokamak device operation and much technical assistance. The authors would also like to acknowledge Prof. L.Chen, Dr F. Zonca, Dr.Z.H.Ling, and Dr.G.Y.Fu for their helpful discussions and assistance on physical analysis. This work is supported by NNSF of China under Grants No. 11005035 and No. 10775004.

\section{References:}

[1] F. Zonca, et al. 2007Nucl. Fusion, 471588.

[2] Furth H.P. 1965 Phys. Fluids 82020

[3] Valoviè M. et al 2000 Nucl. Fusion 401569

[4] Wong K.L. et al 2000 Phys. Rev. Lett. 85996

[5] Ding X.T. et al 2002 Nucl. Fusion 42491

[6] Macor A. et al 2009 Phys. Rev. Lett.102, 155005

[7] Chen W, et al., 2010 Phys. Rev. Lett. 105, 185004

[8] Yang Q.W. et al 2007 Nucl. Fusion 47 S635

[9] Chen W, et.al 2009 Nucl. Fusion 49075022

[10] Chen W, et.al 2010 Nucl. Fusion 50084008

[11] S.C.Jardin, et al., 1986 Journal of computational Physics 66,481 